\title{
CircSAMD4A regulates cell progression and epithelial-mesenchymal transition by sponging miR-342-3p via the regulation of FZD7 expression in osteosarcoma
}

\author{
CHUHAI XIE, BINWEI CHEN, BOYI WU, JIANHONG GUO, YULONG SHI and YANMING CAO \\ Department of Orthopedics, The Second Affiliated Hospital of Guangzhou Medical University, \\ Guangzhou, Guangdong 510260, P.R. China
}

Received August 29, 2019; Accepted January 30, 2020

DOI: $10.3892 / \mathrm{ijmm} .2020 .4585$

\begin{abstract}
Osteosarcoma (OS) is a primary malignant tumor with a complex etiology. Therefore, research into the pathogenesis of osteosarcoma is considered a priority. Circular RNAs play important roles in cell metabolism and in the immune response and are closely associated with cancer treatment. However, research into the association of circular RNAs with osteosarcoma is limited. In the present study, CircSAMD4A was validated by RT-qPCR and agarose gel electrophoresis. CircSAMD4A and miR-342-3p expression was detected by RT-qPCR. The relative protein expression levels were measured by western blot analysis. MTT assay and flow cytometry were used to detect cell cytotoxicity and apoptosis, respectively. Transwell assay was applied to assess cell migration and invasion. Dual-luciferase reporter assay was used to determine the association among CircSAMD4A, Frizzled-7 (FZD7) and miR-342-3p. In vivo, subcutaneous tumor formation assay was performed in an experiment with nude mice. The results revealed that the expression levels of CircSAMD4A and FZD7 were upregulated, while those of miR-342-3p were downregulated in OS tissues and cells. The inhibition of CircSAMD4A suppressed cell progression and epithelial-mesenchymal transition (EMT), and promoted cell apoptosis in OS. The reduction of miR-342-3p reversed the effects of CircSAMD4A downregulation on cell cytotoxicity, migration, invasion, apoptosis and EMT in OS, while FZD7 overexpression blocked the effect of miR-342-3p upregulation on OS progression. The suppressive effect of sh-CircSAMD4A on tumor growth was thus verified in OS. Overall, the present study demonstrated that CircSAMD4A affected cell cytotox-
\end{abstract}

Correspondence to: Dr Chuhai Xie, Department of Orthopedics, The Second Affiliated Hospital of Guangzhou Medical University, 250 Chang-gang-dong Road, Guangzhou, Guangdong 510260, P.R. China

E-mail: xiechuhai@gzhmu.edu.cn

Key words: osteosarcoma, CircSAMD4A, miR-342-3p, Frizzled-7, cell progression, epithelial-mesenchymal transition icity, invasion, apoptosis, migration and EMT by regulating the miR-342-3p/FDZ7 axis in OS, thereby providing a novel regulatory mechanism and a potential therapeutic target for OS.

\section{Introduction}

Osteosarcoma (OS) is a primary malignant tumor that occurs in adolescents and children (1). The pathogenesis of OS is very complex, with surgery and chemotherapy being the main treatment methods $(2,3)$. Moreover, the high recurrence and metastasis of OS patients commonly occurs following standard treatment. Therefore, it is necessary to explore the pathogenesis of OS and to discover novel biomarkers for the targeted treatment of OS.

Circular RNAs are non-coding RNAs with a closed circular structure (4). Due to their insensitivity to nucleases, circular RNAs are more stable and more suitable as diagnostic markers $(5,6)$. Previous studies have demonstrated that circular RNAs play a central regulatory role in the pathogenesis of a variety of cancer types $(7,8)$. Studies have also verified that circular RNAs, as competitive endogenous RNAs, bind to miRNAs and regulate the expression of downstream target genes (9-11). For example, circular RNA circNASP has been shown to regulate OS malignant behavior via the miR-1253/FOXF1 axis (12). CircSAMD4A, a novel circular RNA, was derived from the SAMD4A gene and is located in chr14:55168799-55169298. Yanbin and Jing identified that circSAMD4A was involved in the cellular progression of OS by targeting miR-1244 and regulating MDM2 expression (13). Therefore, the circular RNA-mediated miRNA/mRNA regulatory network plays an essential role in cancer.

MicroRNAs (miRNAs or miRs) are a class of non-coding single-stranded RNAs, ranging from 18 to 22 nucleotides in length, which degrade downstream target genes at the transcriptional and post-transcriptional levels and affect gene expression (14-16). There is accumulating evidence to suggest that miRNAs are widely involved in tumor progression, including cell migration, invasion and proliferation, acting as important regulators in the progression of human diseases, such as hepatocellular carcinoma, osteoarthritis chondrocytes, colorectal cancer and neuroblastoma (17-20). For example, 
miR-503 has been shown to suppress cell proliferation and promote apoptosis by targeting E2F3 in colorectal cancer cells (21). In addition, miRNAs have been considered as biomarkers of cancer treatment and prognosis (22-24). For example, serum miR-542-3p has been verified as a prognostic biomarker in OS (25). miR-221-3p, miR-342-3p and miR-491-3p have been shown to be associated with prognosis in colon cancer (26). miR-21 and miR-29 could thus be used as biomarkers to improve diagnostic efficiency of gastric cancer (27). These data suggest that the regulatory function of miRNAs/mRNAs is critical in the development of various tumors.

The present study demonstrated that CircSAMD4A was highly expressed in OS tissues and cells, suggesting that it plays an important role in the progression of OS. Therefore, biological function assays were performed to further explore the function of CircSAMD4A in OS and to provide novel regulatory mechanisms and therapeutic targets for OS.

\section{Materials and methods}

Tumor specimens and control specimens (pair-matched-adjacent normal tissues) were obtained from 30 patients diagnosed with OS (age, $\geq 25$ years, $n=8$; age, $<25$ years, $n=22$; male/female ratio, 16/14) at the Second Affiliated Hospital of Guangzhou Medical University between August, 2016 and May, 2019. All the patients underwent resection and signed written informed consents. The experiment was approved by the Ethics Committee of the Second Affiliated Hospital of Guangzhou Medical University. All the samples were stored at $-80^{\circ} \mathrm{C}$ until use in the experiments.

Cell culture and transfection. OS cell lines (HOS and U2OS) and the human osteoblast cell line (hFOB 1.19) were purchased from the Chinese Cell Bank of the Chinese Academy of Sciences (Shanghai, China) and cultured in Dulbecco's modified Eagle's medium (DMEM; Gibco; Thermo Fisher Scientific, Inc.) supplemented with 10\% FBS (Gibco; Thermo Fisher Scientific, Inc.), $100 \mathrm{U} / \mathrm{ml}$ of penicillin and $100 \mathrm{mg} / \mathrm{ml}$ of streptomycin (Invitrogen; Thermo Fisher Scientific, Inc.) at $37^{\circ} \mathrm{C}$ in a humidified incubator with $5 \% \mathrm{CO}_{2}$.

Small interfering RNA against CircSAMD4A (si-CircSAMD4A\#1 and si-CircSAMD4A\#2) and negative control si-NC, sh-CircSAMD4A and sh-NC, pcDNA-CircSAMD4A and pcDNA-NC, miR-342-3p mimics (miR-342-3p) and NC, anti-miR-342-3p and anti-NC, pcDNA-FZD7 (FZD7) and vector were purchased from GenePharma. The sequences of si-CircSAMD4A were as follows: (5'-3'): si-CircSAMD4A\#1, AGCACAAGTACAAGAATCATT; si-CircSAMD4A\#2, GCA CAAGTACAAGAATCATTA; miR-342-3p mimic sense, GGG TCTCACACAGAAATCGC and antisense, CAGTGCGTG TCGTGGAGT; miR-342-3p inhibitor, sense, GAGCAGGCT GGAGAA; miRNA mimic negative control sense, UUUGUA CUACACAAAAGUACUG and antisense, CAGUACUUU UGUGUAGUACAAA; miRNA inhibitor negative control sense, CAGUACUUUUGUGUAGUACAAA. Lipofectamine 2000 (Invitrogen; Thermo Fisher Scientific, Inc.) was used to transfect the vectors and oligonucleotides into OS cells for $5 \mathrm{~min}$ at room temperature according to the manufacturer's protocol.
CircRNA expression profile analysis. A gene expression profile (GSE96964) of OS was downloaded from the Gene Expression Omnibus (GEO, https://www.ncbi.nlm.nih.gov/geo/query/acc. cgi?acc=GSE96964). GSE96964 includes 8 samples (U2OS, U2OS/MTX300, HOS, MG63, 143B, ZOS, ZOSM and hFOB1.19 cells). The differentially expressed genes (DEGs) were compared by using GEO2R (https://www.ncbi.nlm.nih. gov/geo/geo2r/?acc=GSE96964).

$R N a s e$ treatment and $R T-q P C R$. Total RNA was isolated from tissues and cells using TRIzol reagent (Invitrogen; Thermo Fisher Scientific, Inc.). The PrimeScript ${ }^{\circledR}$ RT reagent kit (Takara) was applied to reverse transcript RNA to cDNA. The Reverse Transcription Reagents (Applied Biosystems) or TaqMan ${ }^{\circledast}$ MicroRNA Reverse Transcription kit (Applied Biosystems) was used to detect CircSAMD4A and Frizzled-7 (FZD7) or miR-342-3p expression, respectively. Subsequently, qPCR assay was performed on an ABI 7500 Fast Real-Time PCR System (Applied Biosystems) with SYBR ${ }^{\circledR}$ Premix Ex $\mathrm{Taq}^{\mathrm{TM}}$ reagent (Takara). Glyceraldehyde 3-phosphate dehydrogenase (GAPDH) was employed as the normalization gene of CircSAMD4A and FZD7. U6 was employed as the normalization gene of miR-342-3p. The mRNA expression levels of CircSAMD4A and SAMD4A in both HOS and U2OS cell lines were detected by RT-qPCR in the presence or absence of RNase R. The expression of CircSAMD4A, FZD7 and miR-342-3p was calculated using $2^{-\Delta \Delta C t}$ method (28). RT-qPCR was performed under the following thermocycling conditions: $60^{\circ} \mathrm{C}$ for $2 \mathrm{~min}, 95^{\circ} \mathrm{C}$ for $25 \mathrm{sec}$, and 40 circles of $95^{\circ} \mathrm{C}$ for $10 \mathrm{sec}$ and $60^{\circ} \mathrm{C}$ for $30 \mathrm{sec}$. The sequences or the primers were as follows: U6 forward, 5'-CTCGCTTCGGCAGCACA-3' and reverse, 5'-AACGCTTCACGAATTTGCGT-3'; miR-342-3p forward, 5'-TGCGGTCTCACACAGAAATCGCAC-3' and reverse, 5'-CCAGTGCAGGGTCCGAGGT-3'; circSAMD4A forward, 5'-ACTGGCAGGACAAAAGCATG-3' and reverse, 5'-CAGGATTTTGGGCAGCAGTT-3'; FZD7 forward, 5'-TTC TCGGACGATGGCTACC-3' and reverse, 5'-GAACCAAGT GAGAGACAGAATGACC-3'; and GAPDH forward, 5'-GGA GCGAGATCCCTCCAAAAT-3' and reverse, 5'-GGCTGT TGTCATACTTCTCATGG-3'.

Western blot analysis. Total protein was lysed and isolated from cells and tissues using RIPA buffer (Beyotime Institute of Biotechnology) and its quantification was measured using the BCA $^{\mathrm{TM}}$ Protein Assay kit (Pierce; Thermo Fisher Scientific, Inc.), and equal amount proteins ( $20 \mu \mathrm{g})$ were then separated using sodium dodecyl sulfate-polyacrylamide gel electrophoresis (10\% SDS-PAGE). The protein was transferred onto polyvinylidene fluoride (PVDF) membranes (Millipore). The membranes were blocked with PBS containing 5\% non-fat milk for $1 \mathrm{~h}$ at room temperature and then incubated at $4{ }^{\circ} \mathrm{C}$ overnight with the following primary antibodies: FZD7 (1:1,000 dilution, AV41251, Sigma-Aldrich; Merck KGaA), C-caspase-3 (1:2,000 dilution, sc-70497, Santa Cruz Biotechnology, Inc.), N-cadherin (1:2,000 dilution, sc-7939, Santa Cruz Biotechnology, Inc.), proliferating cell nuclear antigen (PCNA; 1:1,000 dilution, MAB424R, Sigma-Aldrich; Merck KGaA), Vimentin (1:2,000 dilution, sc-6260, Santa Cruz Biotechnology, Inc.), E-cadherin (1:2,000 dilution, sc-21791, Santa Cruz Biotechnology, Inc.), Ki-67 (1:1,000 dilution, ab15580, Abcam) and GAPDH 
(1:1,000 dilution, sc-25778, Santa Cruz Biotechnology, Inc.). The membranes were then incubated with horseradish peroxidase (HRP)-conjugated goat anti-rabbit secondary antibody (1:1,000 dilution, sc-2357, Santa Cruz Biotechnology, Inc.) for $1 \mathrm{~h}$ at $37^{\circ} \mathrm{C}$. Clarity Western ECL substrate (Bio-Rad) and ChemiDocTM MP Imaging System (Bio-Rad) were applied to detect the blots and for visualizion. In addition, ImageJ software was used to quantify the blots.

Cell cytotoxicity and apoptosis. The cytotoxicity of the transfected cells was measured using the Cell Growth Determination kit MTT based (Sigma-Aldrich; Merck KGaA). The cells were cultured and seeded into 96 -well plates at a density of $2 \times 10^{3}$ cells per well. Trypsin/EDTA $0.25 \%$ (Sigma-Aldrich; Merck $\mathrm{KGaA}$ ) was then added to each well to generate a single cell suspension. Following incubation for $4 \mathrm{~h}$ at $37^{\circ} \mathrm{C}$ with $5 \%$ of $\mathrm{CO}_{2}, 150 \mu \mathrm{l} \mathrm{MTT}$ solvent $(4 \mathrm{mM} \mathrm{HCl}, 0.1 \% \mathrm{NP} 40$ in isopropanol) was added to each well and incubated at $7^{\circ} \mathrm{C}$ for $3 \mathrm{~h}$ for conversion into formazan. Finally, the optical density (OD) was determined at a wavelength of $450 \mathrm{~nm}$ with a spectrophotometric microplate reader (Beyotime Institute of Biotechnology).

Cell apoptosis was tested using the Annexin V-FITC/PI Apoptosis Detection kit (BD Biosciences) according to the manufacturer'sinstructions. The transfected cellswerecollected and Annexin V-FITC and propidium iodide (PI) were added to incubation at $37^{\circ} \mathrm{C}$ in the dark for $15 \mathrm{~min}$. Cell apoptosis was performed and displayed using flow cytometry.

Cell invasion and migration. A Transwell assay was performed to detect the invasion and migration of the transfected cells. Briefly, the transfected cells $\left(3 \times 10^{3}\right)$ were harvested for $48 \mathrm{~h}$ in serum-free DMEM and then seeded in the upper chambers with $8-\mu \mathrm{m}$ pores coated with Matrigel (BD Biosciences) for invasion assay. For Transwell migration assay, the upper chambers were not coated with Matrigel. An amount of $10 \%$ FBS was added to the medium as a chemoattractant and then the mixed medium was added to the lower chamber. Following incubation for $48 \mathrm{~h}$ at $37^{\circ} \mathrm{C}$, the cells remaining in the upper chambers were removed. The cells attached to the lower surface were stained for $20 \mathrm{~min}$ at $37^{\circ} \mathrm{C}$ using crystal violet. The migrated and invasive cells were detected and counted using a Countess Automatic Cell Counter (Invitrogen; Thermo Fisher Scientific, Inc.).

Animal experiments. A total of 12 female nude mice (6 weeks old; weighing 18-20 g) purchased from Beijing HFK Bioscience Co. Ltd. were used in this study. The mice were housed in a standard animal laboratory where the temperature was maintained at $25^{\circ} \mathrm{C}$ with a humidity level of $40-70 \%$ and 12-h dark/light cycles under pathogen-free conditions. The animals were given free access to food and water. The animal experiments were approved by the Animal Care and Welfare Committee of The Second Affiliated Hospital of Guangzhou Medical University. The mice were divided into 2 groups as follows: sh-CircSAMD4A $(n=6)$ and sh-NC $(n=6)$. Sh-CircSAMD4A and sh-NC $(20 \mu \mathrm{M})$ were transfected into the HOS and U2OS cells and then injected subcutaneously into the right flanks of the female nude mice. The tumor volume was measured every 5 days after the tumors became visible. At 25 days after the injection, all the 12 mice were sacrificed by cervical dislocation that caused a sharp section of the spinal cord followed by an instantaneous cardiac arrest. Subsequently, the tumor weight was detected. The length (L) and width (W) was measured using a vernier caliper. The tumor volume $(\mathrm{V})$ was calculated with the following formula: $\mathrm{V}=\left(\mathrm{LxW}^{2}\right) \times 0.5$.

Dual-luciferase reporter assay. To validate the targeting association among CircSAMD4A, FZD7 and miR-342-3p, a CircSAMD4A-wt, FZD7-wt vector was constructed that contained the matching sequence of miR-342-3p and CircSAMD4A-mut. A FZD7-mut vector was also constructed that contained a mutant sequence. The vector is pmirGLO Dual-Luciferase miRNA Target Expression Vector (Promega). CircSAMD4A-wt or CircSAMD4A-mut and miR-342-3p or NC were then transfected into the U2OS and HOS cells using Lipofectamine 2000 reagent (Invitrogen; Thermo Fisher Scientific, Inc.) according to the manufacturer's instructions. In addition, FZD7-wt or FZD7-mut and miR-342-3p or NC were transfected into the U2OS and HOS cells using Lipofectamine 2000 reagent (Invitrogen; Thermo Fisher Scientific, Inc.). Following transfection for $48 \mathrm{~h}$, the Dual-Luciferase Reporter Assay System (Promega) was used to determine the luciferase activity.

RNA immunoprecipitation (RIP). RIP assay was applied using the EZ-Magna RIP kit (Millipore) according to the manufacturer's protocol. The transfected cells were collected and lysed using RIP buffer, and then incubated with human anti-Argonaute 2 (Ago2) antibody (1:1,000 dilution, ab5072, Abcam) or normal mouse IgG (negative control, 1:1,000 dilution, 12-349, Sigma-Aldrich; Merck KGaA). Following incubation overnight at $4^{\circ} \mathrm{C}$, proteinase $\mathrm{K}$ buffer (Abcam) was added to remove the non-specific binding and the immunoprecipitated RNA was extracted. The purified proteins were detected by RT-qPCR.

Statistical analysis. All data are presented as the means \pm standard deviation (SD). The analysis of the data was performed using GraphPad Prism 7.0 (GraphPad Software). The interaction among CircSAMD4A, miR-342-3p and FZD7 was analyzed by Pearson's correlation analysis. All comparisons were analyzed using Student's t-tests or one-way ANOVA followed by Tukey's test. $\mathrm{P}<0.05$ was considered to indicate a statistically significant difference.

\section{Results}

CircSAMD4A is highly expressed in OS tissues and cell lines. The online data set (GSE96964) revealed that CircSAMD4 (hsa_circRNA_0004846) was highly expressed in the MG-63, U2OS, HOS and 143B cells compared with the hFOB 1.19 cells, suggesting that CircSAMD4 may be associated with OS progression. The present study demonstrated that CircSAMD4 was derived from exon 2 of the host gene SAMD4 according to circBase (http://www.circbase.org/) (Fig. 1A). Divergent primers were then designed to amplify CircSAMD4 and convergent primers wer designed to amplify SAMD4 mRNA. CircSAMD4 was only detectable in cDNA, but not in genomic DNA (gDNA) from the U2OS cells, as shown by RT-qPCR 
A

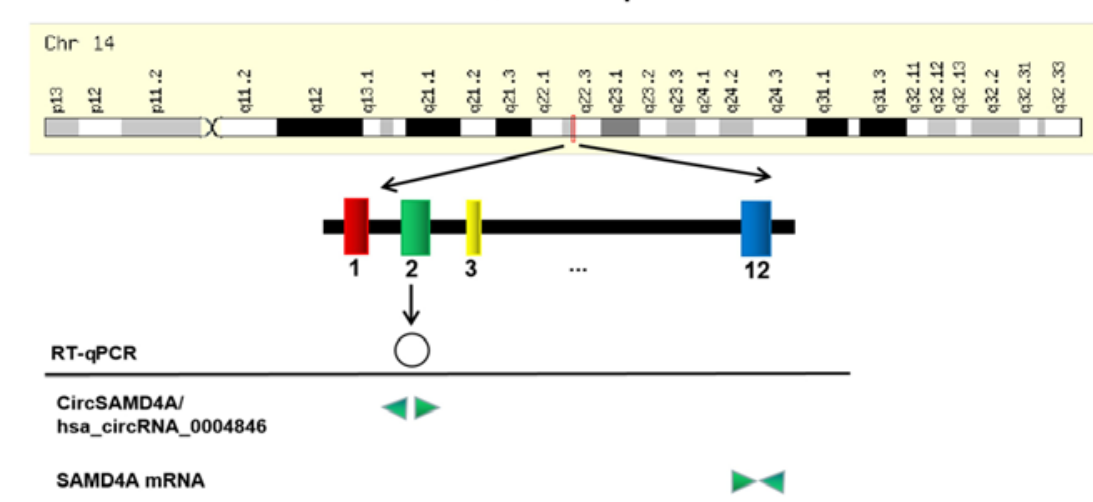

B

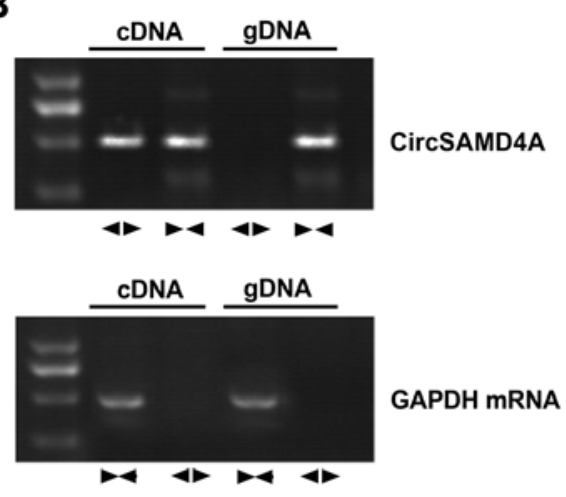

C

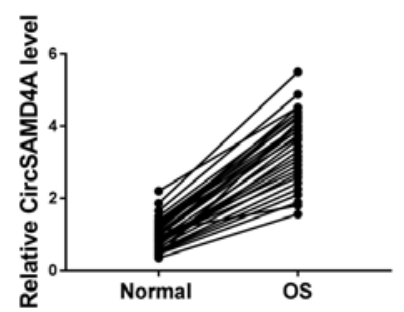

D

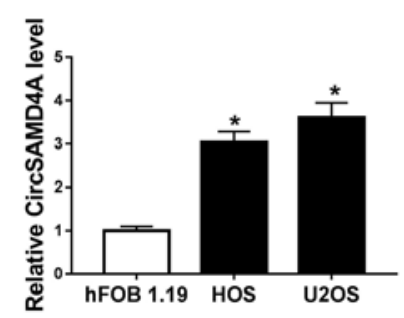

E

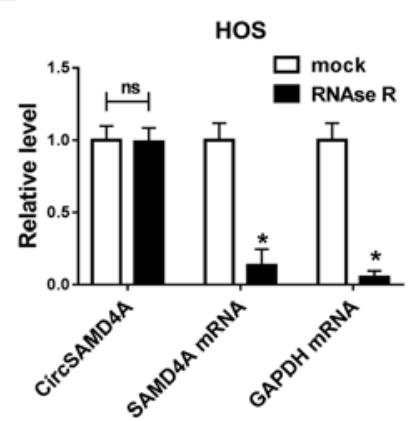

$\mathbf{F}$

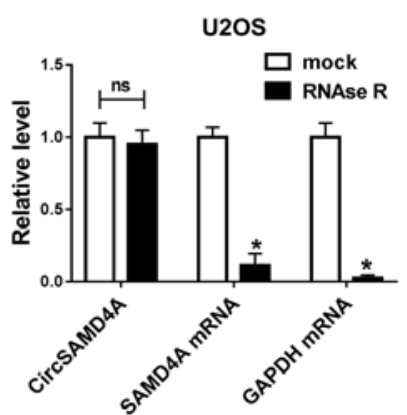

Figure 1. CircSAMD4A is highly expressed in OS tissues and cell lines. (A) CircSAMD4A derived from back-splicing of exon 2 of SAMD4A gene. (B) The existence of CircSAMD4A was detected in U2OS cells by RT-qPCR with convergent or divergent primers and validated by Gel electrophoresis. (C) CircSAMD4A expression was detected in tumor tissues and normal tissues by RT-qPCR. (D) CircSAMD4A expression was detected in OS cell lines (HOS and U2OS) and the human osteoblast cell line (hFOB 1.19) by RT-qPCR. (E and F) CircSAMD4A and SAMD4A mRNA expression in both HOS and U2OS cell lines were detected by RT-qPCR in the presence or absence of RNase R. ${ }^{*}<0.05$, vs. hFOB 1.19 cells or mock; ns, not significant; OS, osteosarcoma.

with divergent primers, while SAMD4 could be amplified in both cDNA and gDNA using convergent primers (Fig. 1B). CircSAMD4A expression was detected in OS tissues and cells. The data demonstrated that CircSAMD4A expression was upregulated in OS tissues compared with normal tissues (Fig. 1C). Additionally, CircSAMD4A expression in the HOS and U2OS cells was significantly higher than that in the hFOB 1.19 cells (Fig. 1D). Moreover, RNase R assay was applied to confirm the stability of CircSAMD4A. The results revealed that the expression of the linear forms of SAMD4A markedly decreased following RNase R treatment; however, RNase R failed to digest CircSAMD4A in the HOS and U2OS cells (Fig. 1E and F). Therefore, CircSAMD4A was upregulated in OS cells and tissues, indicating that CircSAMD4A is associated with OS progression.

Knockdown of CircSAMD4A promotes cytotoxicity and apoptosis, and inhibits the migration, invasion and epithelial-mesenchymal transition (EMT) of OS cells. To further examine the effects of CircSAMD4A on OS progression, si-NC and si-CircSAMD4A were transfected into the HOS and U2OS cells. The results indicated that CircSAMD4A was inhibited in the HOS and U2OS cells, which were transfected with si-CircSAMD4A, and 2 transfected cell lines (si-CircSAMD4A\#1 and si-CircSAMD4A\#2) were selected for use in the following experiments (Fig. 2A). As presented in Fig. 2B, MTT assay suggested that cell cytotoxicity was enhanced by CircSAMD4A downregulation. Moreover,
Ki-67 protein expression was decreased by si-CircSAMD4A transfection (Fig. 2C). The knockdown of CircSAMD4A suppressed OS cell growth. Flow cytometry demonstrated that CircSAMD4A downregulation contributed to OS cell apoptosis (Fig. 2D and E). The protein level of C-caspase 3 was markedly induced by the knockdown of CircSAMD4A (Fig. 2F). Transwell assay confirmed that the migration and invasion of the HOS and U2OS cells were decreased by the knockdown CircSAMD4A (Fig. 2G and H). Furthermore, the knockdown of CircSAMD4A inhibited N-cadherin and Vimentin protein expression, whereas it increased E-cadherin protein expression in the HOS and U2OS cells (Fig. 2I and J). These data demonstrated that the knockdown of CircSAMD4A suppressed the migration, invasion and EMT of OS cells, and promoted the cytotoxicity and apoptosis of OS cells, suggesting that CircSAMD4A plays a crucial role in OS progression.

Knockdown of CircSAMD4A suppresses tumor growth in vivo. Subsequently, the function of CircSAMD4A was verified in a mouse experiment. sh-NC and sh-CircSAMD4A were transfected into U2OS cells and the transfected cells were then injected into mice. The results revealed that the tumor volume in the CircSAMD4A knockdown group was significantly smaller than that of the sh-NC group (Fig. 3A). Similarly, CircSAMD4A knockdown significantly inhibited tumor weight (Fig. 3B). In the tumor tissues, the expression of CircSAMD4A was significantly reduced by sh-CircSAMD4A (Fig. 3C). Moreover, compared with the sh-NC group, PCNA 
B

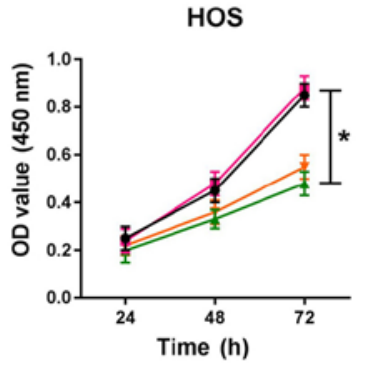

A

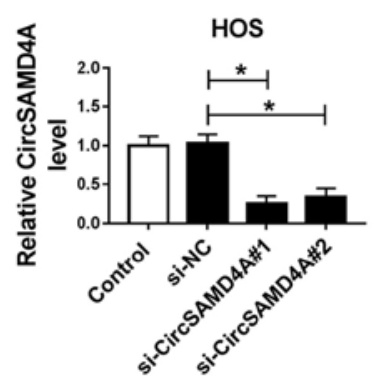

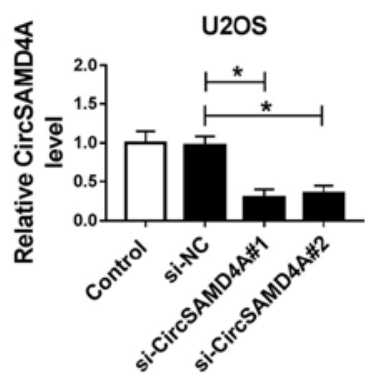

C

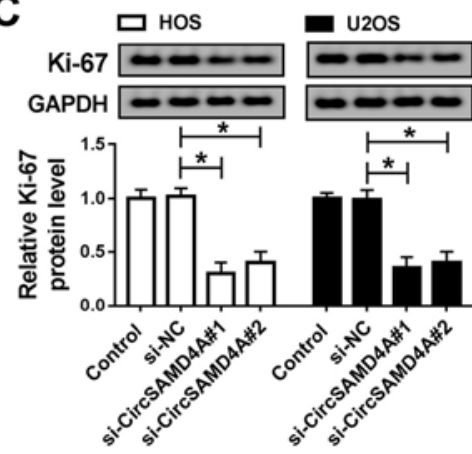

D

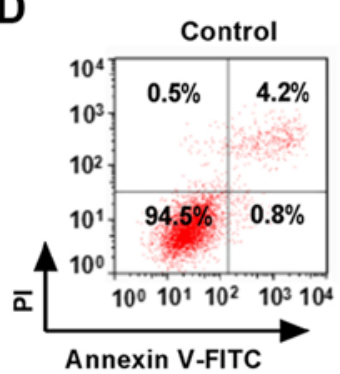

$\rightarrow$ Control

- si-NC

* si-CircSAMD4A\#1

* si-CircSAMD4A\#2
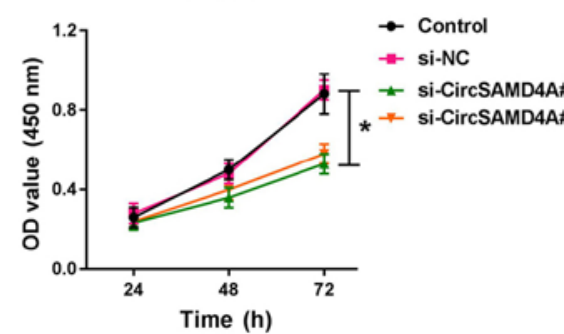

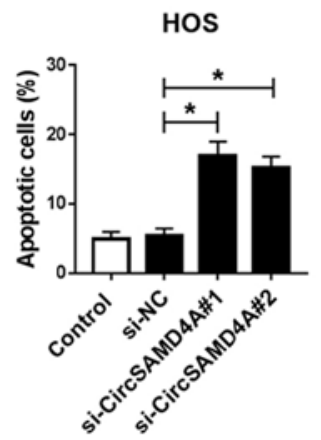

U2OS

E

Control
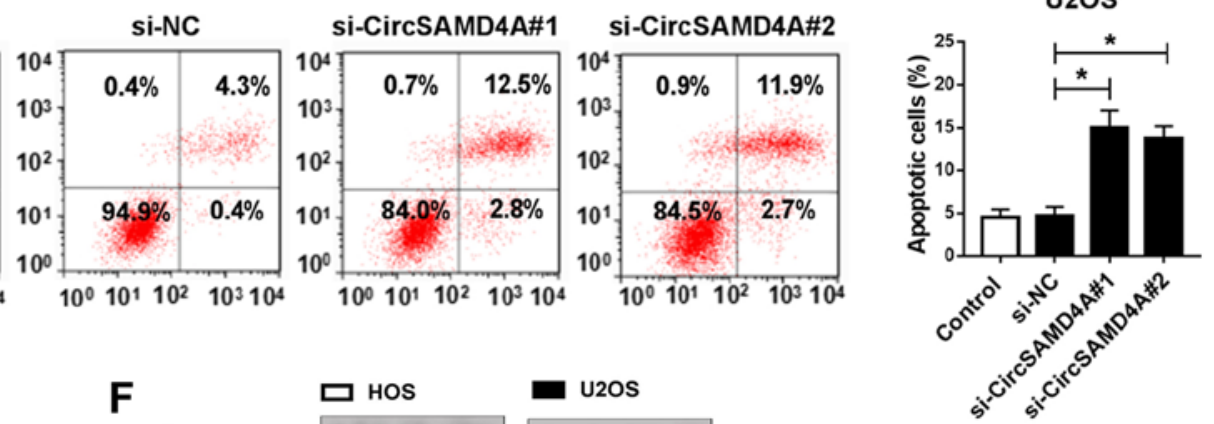

F

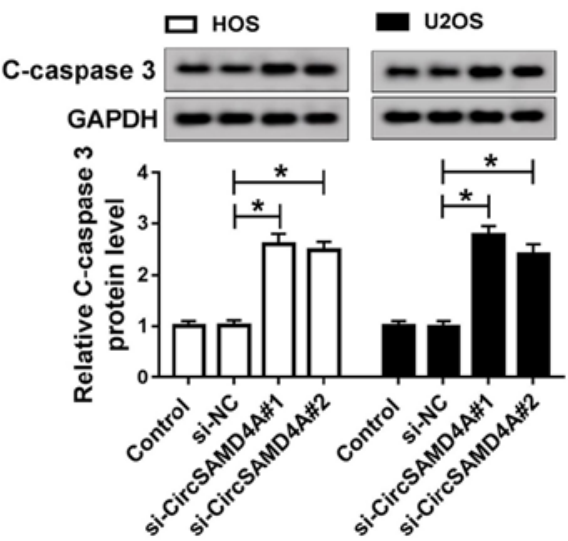

Figure 2. Knockdown of CircSAMD4A inhibits the migration, invasion and EMT, and promotes the cytotoxicity and apoptosis of OS cells. (A) CircSAMD4A expression of control, si-NC, si-CircSAMD4A\#1 or si-CircSAMD4A\#2 groups was detected by RT-qPCR. (B) MTT assay was applied to measure the cytotoxicity of HOS and U2OS cells transfected with control, si-NC si-CircSAMD4A\#1 and si-CircSAMD4A\#2. (C) The protein expression of Ki-67 was measured in control, si-NC, si-CircSAMD4A\#1 and si-CircSAMD4A\#2 groups in HOS and U2OS cells by western blot analysis. (D and E) Cell apoptosis of control, si-NC si-CircSAMD4A\#1 and si-CircSAMD4A\#2 groups detected by flow cytometry in HOS and U2OS cells. (F) The protein expression of C-caspase-3 was detected in control, si-NC si-CircSAMD4A\#1 and si-CircSAMD4A\#2 groups in HOS and U2OS cells by western blot analysis. 

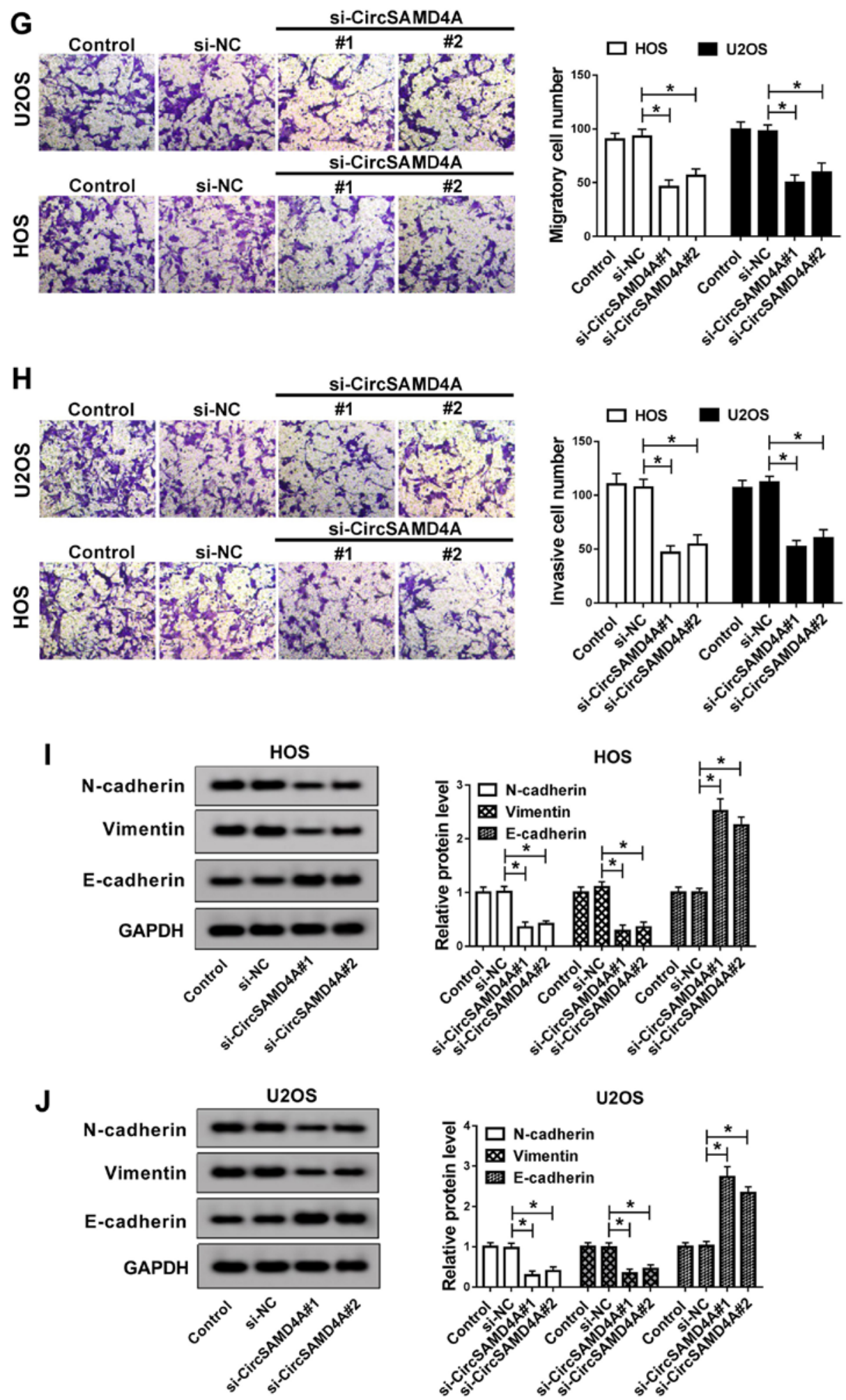

Figure 2. Continued. (G and $\mathrm{H}$ ) Cell migration and invasion of control, si-NC si-CircSAMD4A\#1 and si-CircSAMD4A\#2 groups were detected using Transwell assay in HOS and U2OS cells. (I and J) The protein expression of N-cadherin, Vimentin and E-cadherin of control, si-NC si-CircSAMD4A\#1 and si-CircSAMD4A\#2 groups was measured by western blot analysis in HOS and U2OS cells. "P<0.05, vs. respective control. OS, osteosarcoma; EMT, epithelial-mesenchymal transition.

protein expression was markedly reduced by CircSAMD4A downregulation, while the protein expression of C-caspase-3 was markedly increased (Fig. 3D). Moreover, the protein expression of $\mathrm{N}$-cadherin and Vimentin in the sh-CircSAMD4A group was significantly lower than that in the sh-NC group, while the protein expression of E-cadherin was markedly higher than that in the sh-NC group (Fig. 3E). These results revealed that the downregulation of CircSAMD4A significantly inhibited the tumor growth of OS in vivo.

miR-342-3p expression is inhibited in OS tissues and cells, and it is a target miRNA of CircSAMD4A in OS. Based on 
A

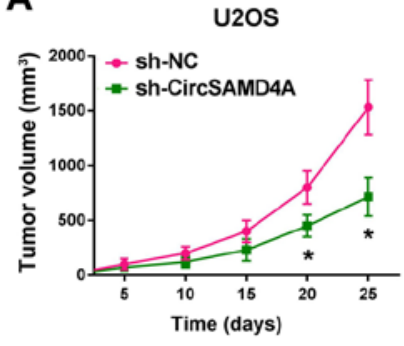

D

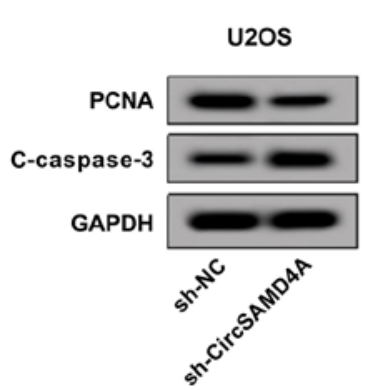

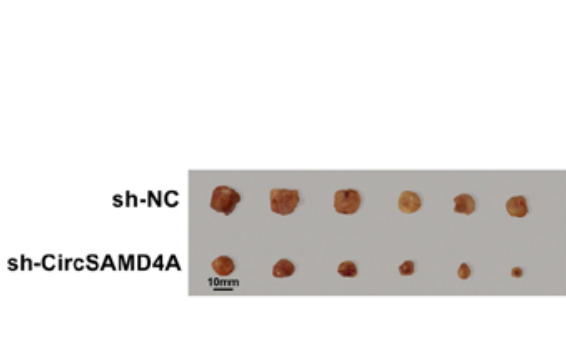

B

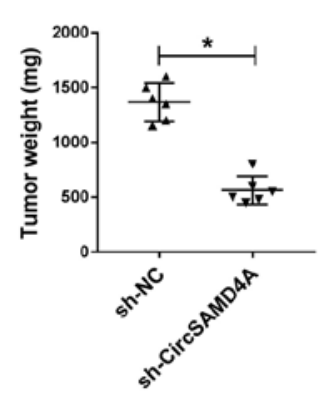

C

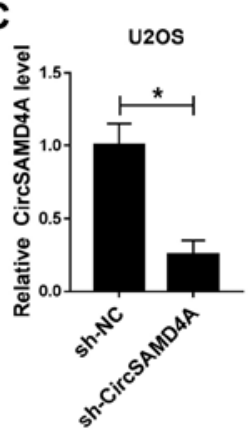

U2OS

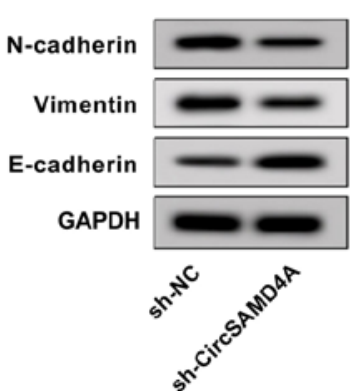

U2OS

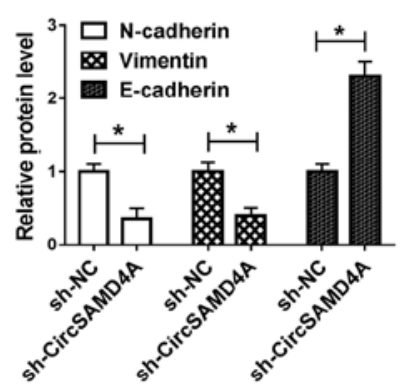

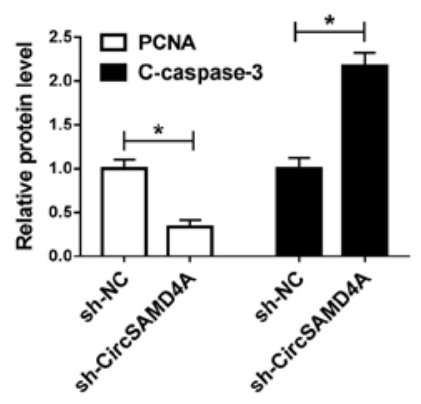

Figure 3. Knockdown of CircSAMD4A suppresses tumor growth in vivo. (A and B) Tumor volume and weight were calculated in vivo. (C) CircSAMD4A expression was inhibited by sh-CircSAMD4A transfection, as shown by RT-qPCR. (D and E) The protein expression of PCNA, C-caspase-3, N-cadherin, Vimentin and E-cadherin was detected in sh-NC and sh-CircSAMD4A groups. " $\mathrm{P}<0.05$, vs. sh-NC.

A

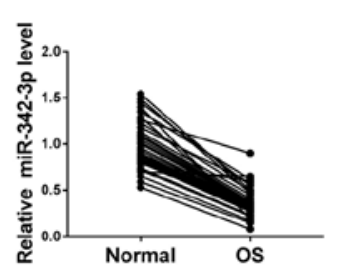

E

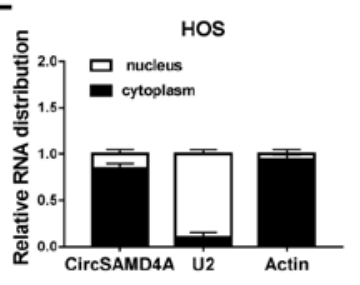

G

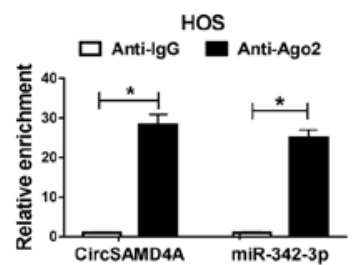

B
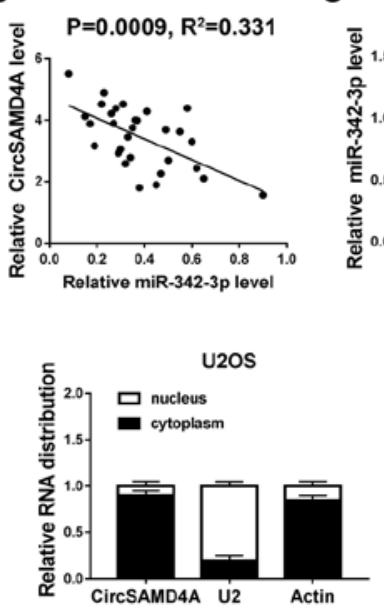

U2OS

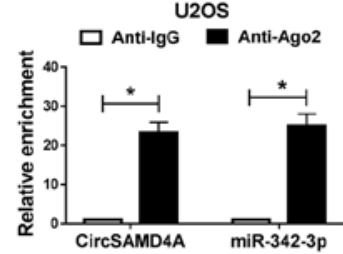

C

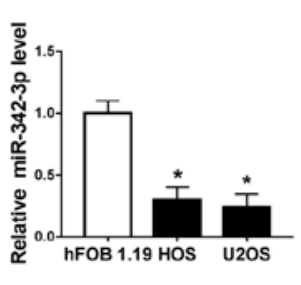

$\mathbf{F}$

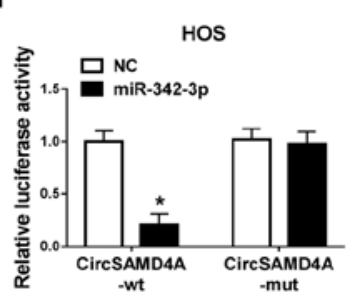

H

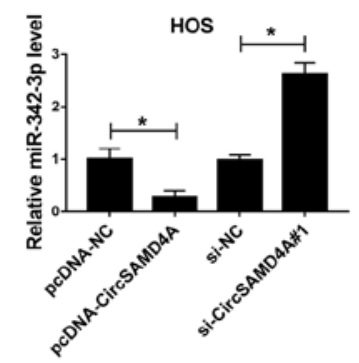

D

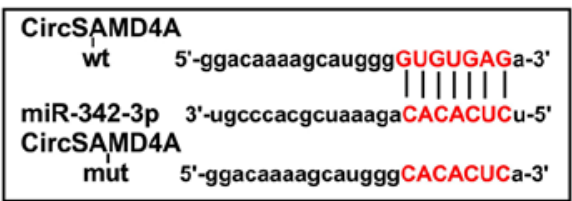

Figure 4. miR-342-3p expression is decreased in OS tissues and cells, and miR-342-3p is a target miRNA of CircSAMD4A knockdown in OS. (A) miR-342-3p expression was detected in tumor tissues and normal tissues by RT-qPCR. (B) Pearson's correlation analysis was used to verify the relationship between miR-342-3p and CircSAMD4A in tumor tissues. (C) miR-342-3p expression was detected in OS cell lines (HOS and U2OS) and the human osteoblast cell line (hFOB 1.19) by RT-qPCR. (D) Putative sequences of miR-342-3p and CircSAMD4A. (E) CircSAMD4A expression in nucleus and cytoplasm in HOS and U2OS cells was detected by RT-qPCR using Actin as marker in the cytoplasm and U2 as a marker to identify the nuclear compartment. (F) Dual-luciferase reporter assay was applied to measure the luciferase activity of HOS and U2OS cells transfected with miR-342-3p or NC and CircSAMD4A-wt or CircSAMD4A-mut. (G) CircSAMD4A and mir-342-3p expression bound to Ago 2 antibody was detected by RIP assay. (H) miR-342-3p expression was detected in pcDNA-NC, pcDNA-CircSAMD4A, si-NC and si-CircSAMD4A groups by RT-qPCR in HOS and U2OS cells. " $\mathrm{P}<0.05$, vs. respective control. OS, osteosarcoma. 

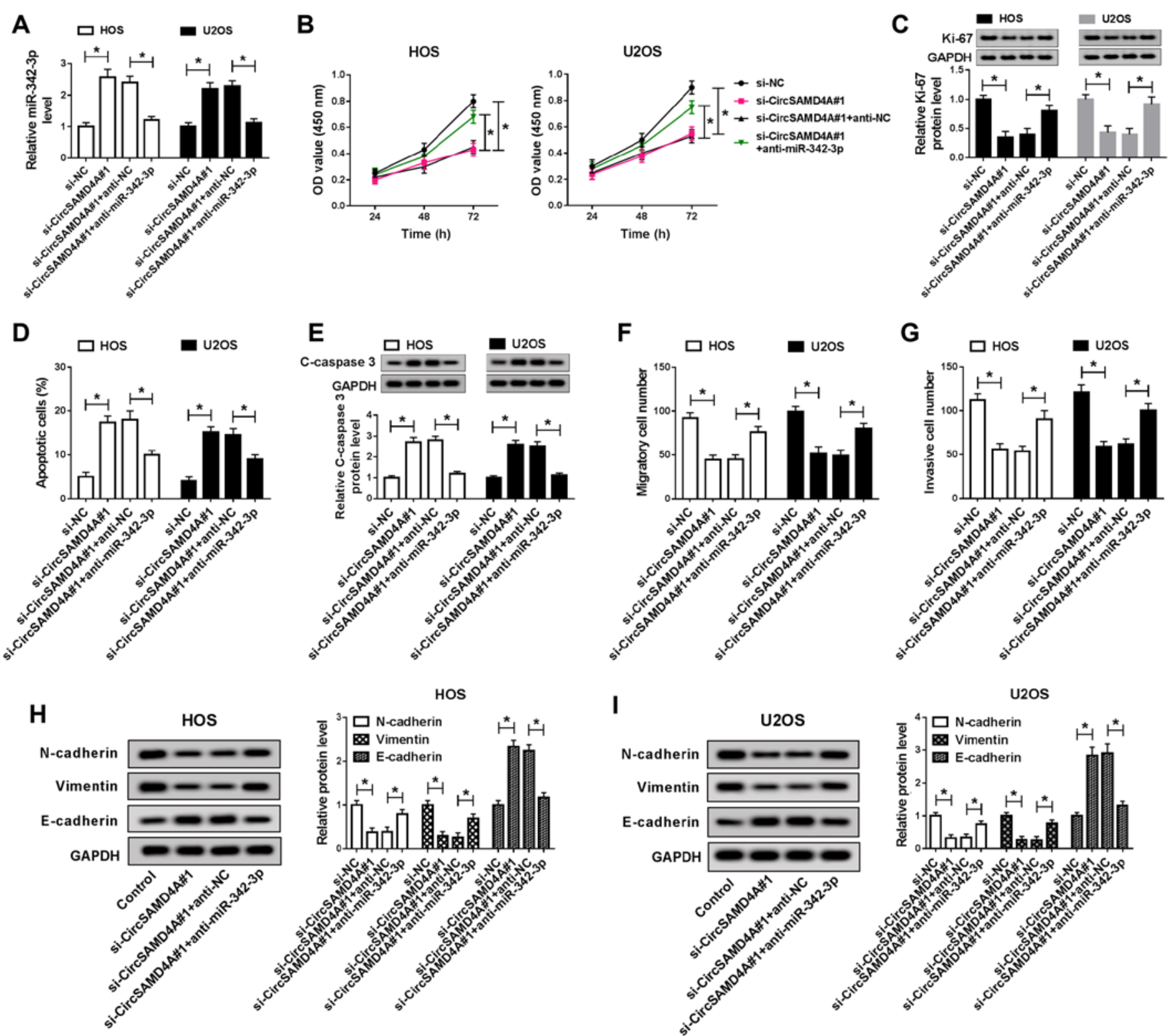

Figure 5. Inhibition of miR-342-3p reverses the suppressive effects of si-CircSAMD4A on the proliferation and EMT of OS cells. (A) CircSAMD4A expression of si-NC, si-CircSAMD4A\#1, si-CircSAMD4A\#1+anti-NC and si-CircSAMD4A\#1+anti-miR-342-3p groups was detected by RT-qPCR in HOS and U2OS cells. (B) MTT assay was applied to measure the cytotoxicity of HOS and U2OS cells transfected with si-NC, si-CircSAMD4A, si-CircSAMD4A+anti-NC and si-CircSAMD4Atanti-miR-342-3p. (C) The protein expression of Ki-67 was detected in si-NC, si-CircSAMD4A\#1, si-CircSAMD4A\#1+anti-NC and si-CircSAMD4A\#1+anti-miR-342-3p groups by western blot analysis. (D) Cell apoptosis of si-NC, si-CircSAMD4A\#1, si-CircSAMD4A\#1+anti-NC and si-CircSAMD4A\#1+anti-miR-342-3p groups was detected by flow cytometry in HOS and U2OS cells. (E) The protein of C-caspase-3 was detected in si-NC, si-CircSAMD4A\#1, si-CircSAMD4A\#1+anti-NC and si-CircSAMD4A\#1+anti-miR-342-3p groups in HOS and U2OS cells by western blot analysis. ( $\mathrm{F}$ and $\mathrm{G}$ ) Cell migration and invasion of si-NC, si-CircSAMD4A\#1, si-CircSAMD4A\#1+anti-NC and si-CircSAMD4A\#1+anti-miR-342-3p groups was detected using Transwell assay in HOS and U2OS cells. (H and I) The protein expression of N-cadherin, Vimentin and E-cadherin of si-NC, si-CircSAMD4A\#1, si-CircSAMD4A\#1+anti-NC and si-CircSAMD4A\#1+anti-miR-342-3p groups was measured by western blot analysis in HOS and U2OS cells. "P<0.05, vs. respective control. OS, osteosarcoma; EMT, epithelial-mesenchymal transition.

bioinformatics analysis, the present study demonstrated that miR-342-3p contained a binding site of CircSAMD4A, predicting that miR-342-3p is a candidate target miRNA of CircSAMD4A (Fig. 4D). Thus, miR-342-3p expression was subsequently detected in OS tissues and cells. The data revealed that miR-342-3p expression was significantly decreased in OS tissues and cells, and that the miR-342-3p level negatively correlated with CircSAMD4A expression in OS (Fig. 4A-C). Subsequently, nuclear and cytoplasmic separation experiments were performed using actin as a marker in the cytoplasm and U2 as a marker to identify the nuclear compartment, in order to determine the subcellular localization of CircSAMD4A in the nucleus and cytoplasm. The results indicated that the expression of CircSAMD4A in the cytoplasm was significantly higher than that in the nucleus in the HOS and U2OS cells (Fig. 4E). In addition, the dual-luciferase reporter assay demonstrated that the luciferase activity was significantly decreased when miR-342-3p was bound to CircSAMD4A-wt; however, there was no significant change in the luciferase activity when miR-342-3p was bound to CircSAMD4A-mut in the HOS and U2OS cells (Fig. 4F). The experimental combination of RIP also demonstrated that 
A

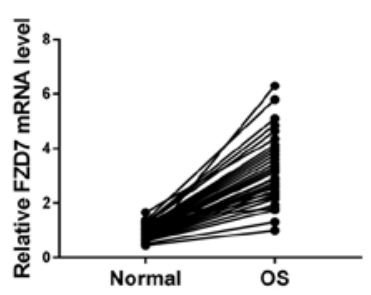

E
B

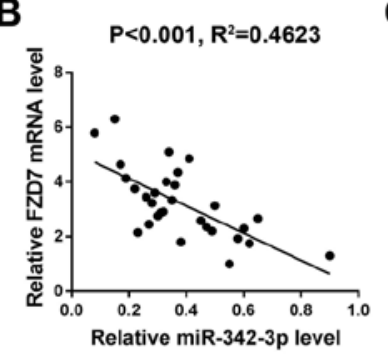

C

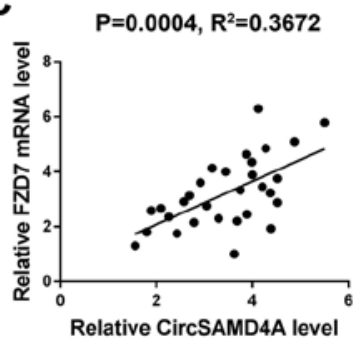

F

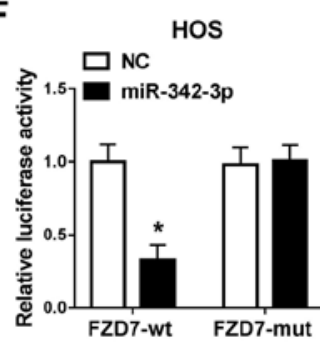

H

G
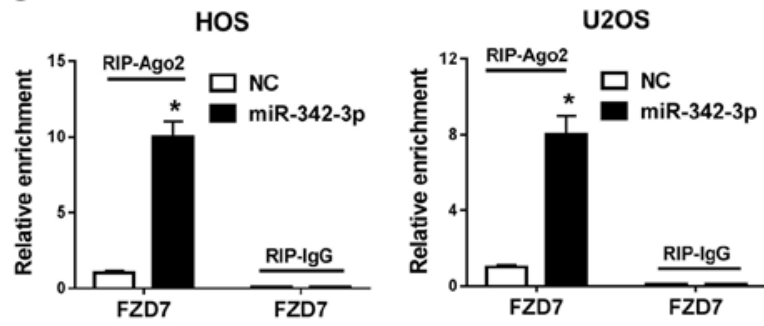

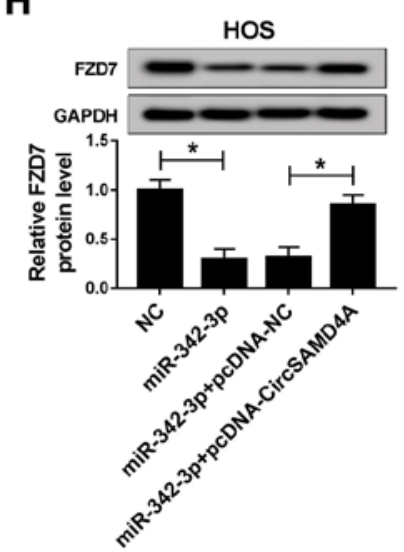

D
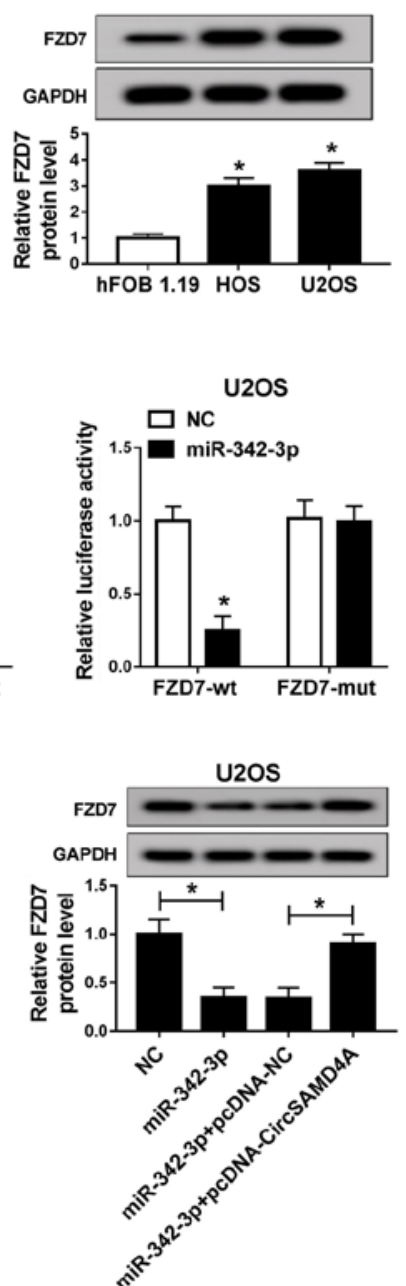

Figure 6. FZD7 is a direct target gene of miR-342-3p in OS. (A) FZD7 expression was detected in tumor tissues and normal tissues using qRT-PCR. (B and C) Pearson's correlation analysis was used to verify the correlation among CircSAMD4A, miR-342-3p and FZD7 in tumor tissues. (D) FZD7 expression was detected in OS cell lines (HOS and U2OS) and human osteoblast cell line (hFOB 1.19) by RT-qPCR. (E) The putative sequences of miR-342-3p and FZD7. (F) Dual-luciferase reporter assay was applied to measure the luciferase activity of HOS and U2OS cells transfected with miR-342-3p or NC and FZD7-wt or FZD7-mut. (G) FZD7 and mir-342-3p expression bound to Ago 2 antibody was detected by RIP assay. (H) FZD7 expression in NC, miR-342-3p, miR-342-3p+pcDNA-NC and miR-342-3p+pcDNA-CircSAMD4A groups was detected by RT-qPCR in HOS and U2OS cells. "P<0.05, vs. respective control. OS, osteosarcoma; FZD7, frizzled family receptor 7.

miR-342-3p is the target miRNA of CircSAMD4A in HOS and U2OS cells (Fig. 4G). Moreover, miR-342-3p expression in the pcDNA-NC, pcDNA-CircSAMD4A, si-NC and si-CircSAMD4A groups was determined. The results revealed that CircSAMD4A overexpression suppressed the expression level of miR-342-3p, while CircSAMD4A knockdown promoted the miR-342-3p level in the HOS and U2SO cells (Fig. 4H). These data indicated that miR-342-3p was the target miRNA of CircSAMD4A in OS.

Inhibition of miR-342-3p reverses the suppressive effects of si-CircSAMD4A on cell progression and EMT in OS cells. To further clarify the function of CircSAMD4A and miR-342-3p regulatory networks in OS cell progression, rescue experiments were conducted. The HOS and U2OS cells were transfected with si-NC, si-CircSAMD4A\#1, si-CircSAMD4A\#1+anti-NC or si-CircSAMD4A\#1+anti-miR -342-3p. miR-342-3p expression was significantly induced by the knockdown of CircSAMD4A; however, miR-342-3p inhib- itor attenuated this effect (Fig. 5A). In addition, the results of MTT assay and Transwell assay revealed that the knockdown of CircSAMD4A significantly promoted cell cytotoxicity and inhibited cell migration and invasion, while these effects were impaired by miR-342-3p inhibition (Fig. 5B, F and G). Moreover, reducing CircSAMD4A expression significantly increased OS cells apoptosis, while this promotion effect was significantly harbored by miR-342-3p downregulation (Fig. 5D). The results of western blot analysis revealed that the knockdown of CircSAMD4A promoted the expression of C-caspase- 3 and E-cadherin, and inhibited the expression of Ki-67, N-cadherin and Vimentin; these effects were reversed by the inhibition of miR-342-3p (Fig. 5C, E, H and I). Overall, these findings demonstrate that CircSAMD4A regulates OS cell progression by targeting miR-342-3p.

FZD7 is a direct target gene of miR-342-3p in OS. It is well known that miRNAs can regulate the downstream target genes to affect cell growth. Moreover, FZD7, a Wnt signaling 
A

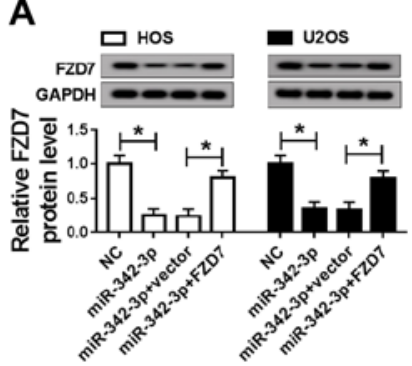

D

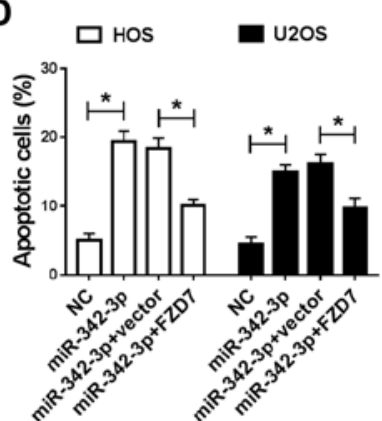

H

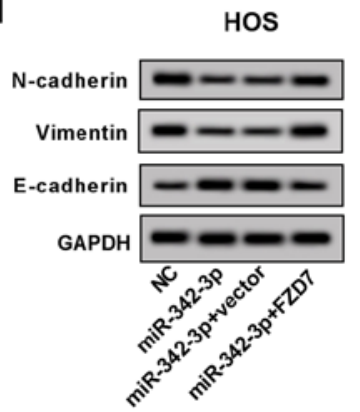

B

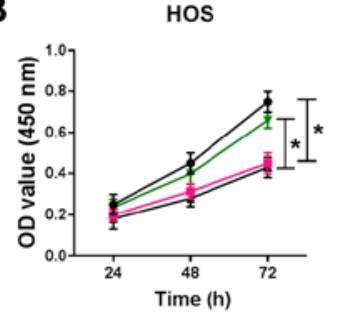

E
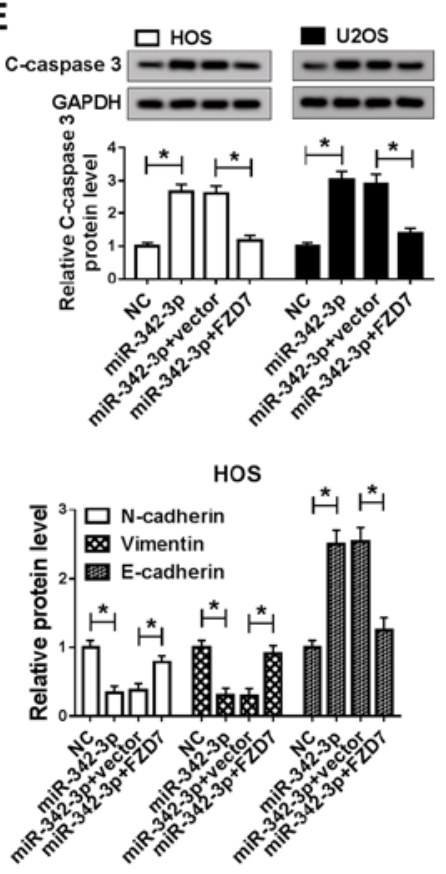

U20S

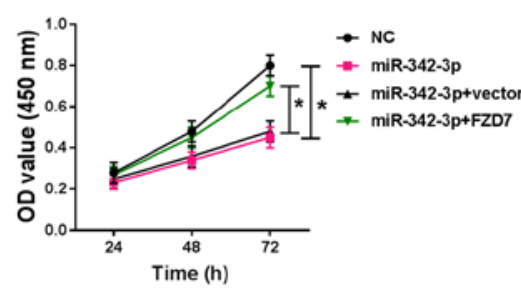

$\mathbf{F}$

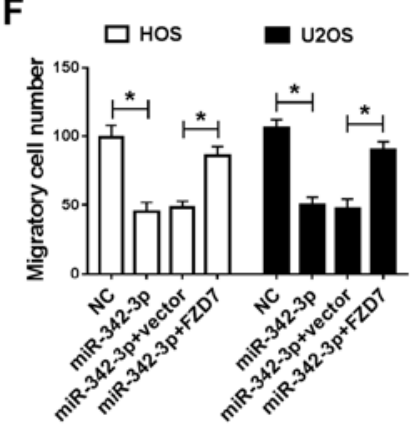

I

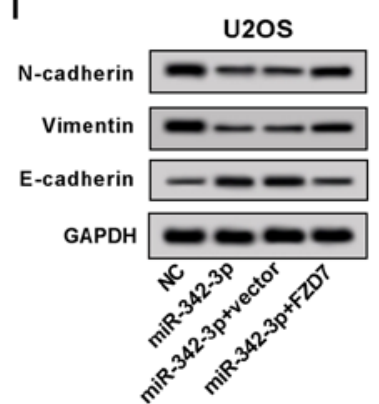

C

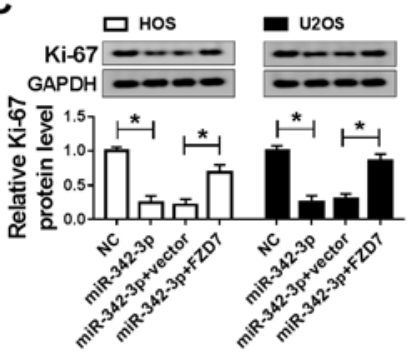

G
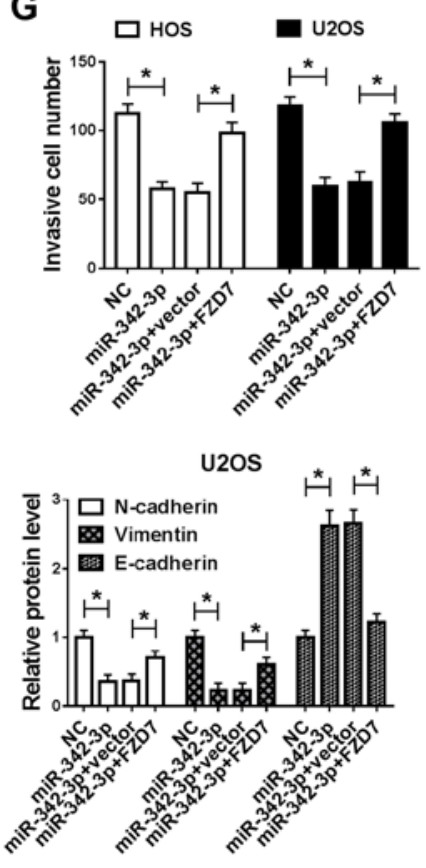

Figure 7. The promotion of FZD7 attenuates the suppressive effects of miR-342-3p overexpression on the proliferation and EMT of OS cells. (A) FZD7 expression in NC, miR-342-3p, miR-342-3p+vector and miR-342-3p+FZD7 groups in HOS and U2OS cells was detected by RT-qPCR. (B) MTT assay was applied to measure the cytotoxicity of HOS and U2OS cells transfected with NC, miR-342-3p, miR-342-3p+vector and miR-342-3p+FZD7. (C) Ki-67 protein expression was detected in NC, miR-342-3p, miR-342-3p+vector and miR-342-3p+FZD7 groups in HOS and U2OS cells by western blot analysis. (D) Cell apoptosis was measured by flow cytometry in NC, miR-342-3p, miR-342-3p+vector and miR-342-3p+FZD7 groups in HOS and U2OS cells. (E) The protein of C-caspase 3 was detected in NC, miR-342-3p, miR-342-3p+vector and miR-342-3p+FZD7 groups in HOS and U2OS cells by western blot analysis. (F and G) Cell migration and invasion of NC, miR-342-3p, miR-342-3p+vector and miR-342-3p+FZD7groups was detected using Transwell assay in HOS and U2OS cells. (H and I) The protein expression of N-cadherin, Vimentin and E-cadherin of NC, miR-342-3p, miR-342-3p+vector and miR-342-3p+FZD7 groups was measured by western blot analysis in HOS and U2OS cells. ${ }^{*} \mathrm{P}<0.05$, vs. respective control. OS, osteosarcoma; EMT, epithelial-mesenchymal transition.

receptor, has been confirmed as a carcinogenic factor in OS (29). The present study demonstrated that FZD7 was a potential target gene of miR-342-3p, with its 3'UTR containing the complement of miR-342-3p (Fig. 6E). In addition, it was found that FZD7 was highly expressed in OS tissues and cells (Fig. 6A and D). As illustrated in Fig. 6B and C, FZD7 expression negatively correlated with the miR-342-3p level and positively correlated with CircSAMD4A expression. Moreover, the luciferase reporter assay demonstrated that when miR-342-3p was bound to FZD7-wt 3'UTR, the luciferase activity was significantly decreased (Fig. 6F). RIP assay revealed that FZD7 was enriched in the miR-342-3p groups (Fig. 6G). These results indicated that FZD7 was the target gene of miR-342-3p in the HOS and U2OS cells. In addition, FZD7 expression in the HOS and U2OS was significantly inhibited by miR-342-3p overexpression, while CircSAMD4A overexpression reversed the inhibitory effect of miR-342-3p on FZD7 expression (Fig. 6H). In summary, these results indicated that FZD7 was a target gene of miR-342-3p.
The promotion of FZD7 attenuates the suppressive effects of miR-342-3p overexpression on cell progression and EMT in OS cells. To verify the effect of miR-342-3p and FDZ7 regulatory networks on OS cells, recovery experiments were conducted. As illustrated in Fig. 7A, miR-342-3p overexpression inhibited FZD7 expression, and this effect was impaired by increasing FZD7 expression in HOS and U2OS cells. Furthermore, increasing miR-342-3p expression significantly inhibited the migration and invasion, and increased the cytotoxicity and apoptosis of HOS and U2OS cells, while these effects were attenuated by FZD7 overexpression (Fig. 7B, D, F and G). Notably, miR-342-3p overexpression induced an increase in C-caspase-3, E-cadherin protein expression, and a decrease in $\mathrm{Ki}-67$, $\mathrm{N}$-cadherin and Vimentin protein expression; however, these effects of miR-342-3p overexpression were reversed by FZD7 upregulation (Fig. 7C, E, H and I). Taken together, these findings demonstrate that miR-342-3p regulates OS cell growth and EMT by targeting FZD7. 


\section{Discussion}

The diagnosis and treatment of OS have always been a main concern for researchers. The advancement of biotechnology and the development of next-generation sequencing technology are likely to provide target therapy for OS. Cell proliferation, migration, and invasion are essential phenomena with respect to cancer cell metastasis and development. EMT is important in cancer metastasis and is closely associated with cancer cell migration, invasion and apoptosis. N-cadherin, E-cadherin and Vimentin proteins are important markers in the cellular EMT mechanism, which can reflect the degree of EMT in cells (30).

Cyclic RNAs have been found to be involved in tumor progression in various cancer types. Circular RNAs have been confirmed to exert various effects, including functioning as biomarkers to improve the diagnostic efficiency and serving as a regulatory factor in OS progression (12,31-33). In the study by Liu et al, it was reported that circNT52 functioned as an oncogene to regulate OS cell proliferation and metastasis by sponging miR-448 (34). CircSAMD4A has been reported to be highly expressed in OS and to regulate cell proliferation by targeting the miR-1244/MDM2 axis (27). In this study, CircSAMD4A was identified to exhibit a significant high expression in OS tissues and cells. CircSAMD4A deletion significantly attenuated the ability of cell metastasis, and promoted cytotoxicity and apoptosis in OS in vitro. In addition, the suppressive effect of CircSAMD4A knockdown on OS tumor growth was confirmed in an experiment on nude mice. These data proved that the downregulation of CircSAMD4A suppressed OS progression in vitro and in vivo. According to the results, CircSAMD4A expression was increased in OS tissues and cells, and CircSAMD4A silencing inhibited proliferation in OS (27). Moreover, the present study revealed that miR-342-3p was a target miRNA of CircSAMD4A. miR-342-3p has been demonstrated to be involved in cellular growth in cancers, including pancreatic cancer, hepatocellular carcinoma, prostate cancer and cervical cancer (35-38). Zhang et al reported that miR-342-3p was downregulated in OS tissues and inhibited cell progression by targeting AEG-1 (39). However, the regulatory mechanism of miR-342-3p in OS has not been fully elucidated. The present study revealed that miR-342-3p expression was decreased in OS, a finding which is in accordance with the previous study by Zhang et al (39). miR-342-3p overexpression inhibited OS cell growth and metastasis. Moreover, the inhibition of miR-342-3p reversed the inhibitory effects of CircSAMD4A deletion on OS cell progression. Thus, CircSAMD4A affected OS cell growth and EMT by modulating miR-342-3p.

Finally, the present study also demonstrated that FZD7 was a target gene of miR-342-3p. A number of studies have indicated that FZD7 is an important regulator in the cellular mechanism of cancers (40-42). For example, FZD7 overexpression can induce cell proliferation in glioma (43). Moreover, FZD7 has been verified to be a novel prognostic marker and ti contribute to the regulation of tumor metastasis (44). In addition, FZD7 has also been shown to be associated with resistance and prognosis $(45,46)$. This study revealed that FZD7 was upregulated in OS tissues and cells. The accumulation of FDZ7 reversed the inhibitory effects of miR-342-3p overexpression on cell growth and metastasis in OS, suggesting that CircSAMD4A regulates OS progression by sponging miR-342-3p to modulate FDZ7 expression.

In conclusion, the present study demonstrated that CircSAMD4A affected cell cytotoxicity, invasion, apoptosis, migration and EMT by regulating the miR-342-3p/FDZ7 axis in OS, thereby providing a novel regulatory mechanism of OS and a potential therapeutic target for OS.

\section{Acknowledgements}

Not applicable.

\section{Funding}

This study was supported by the National Natural Science Foundation of China (81574002).

\section{Availability of data and materials}

All data generated or analyzed during this study are included in this published article or are available from the corresponding author on reasonable request.

\section{Authors' contributions}

CX was responsible for the conception and design of the study. $\mathrm{BC}$ was involved in the development of the study methodology. BW was involved in data acquisition. JG was involved in the analysis and interpretation of the data. YS was involved in the writing, reviewing and revision of the article and analyzed the literature, and interpreted the data. YC was involved in providing administrative, technical and material support, analyzed the literature, interpreted the data and produced the figures. All authors have reviewed and approved of the article prior to submission and have read and approved the final article.

\section{Ethics approval and consent to participate}

All patients underwent resection and signed written informed consents. The use of patient samples was approved by the Ethics Committee of the Second Affiliated Hospital of Guangzhou Medical University. Animal experiments were approved by the Animal Care and Welfare Committee of The Second Affiliated Hospital of Guangzhou Medical University.

\section{Patient consent for publication}

Not applicable.

\section{Competing interests}

The authors declare that they have no competing interests.

\section{References}

1. Meyers PA and Gorlick R: Osteosarcoma. Pediatr Clin North Am 44: 973-989, 1997.

2. Longhi A, Errani C, De Paolis M, Mercuri M and Bacci G: Primary bone osteosarcoma in the pediatric age: State of the art. Cancer Treat Rev 32: 423-436, 2006. 
3. Kempfbielack B, Bielack SS, Jürgens H, Branscheid D Berdel WE, Exner GU, Göbel U, Helmke K, Jundt G, Kabisch H, et al: Osteosarcoma relapse after combined modality therapy: An analysis of unselected patients in the cooperative osteosarcoma study group (COSS). J Clin Oncol 23: 559-568, 2005.

4. Sanger HL, Klotz G, Riesner D, Gross HJ and Kleinschmidt AK: Viroids are single-stranded covalently closed circular RNA molecules existing as highly base-paired rod-like structures. Proc Natl Acad Sci USA 73: 3852-3856, 1976.

5. Guo JU, Agarwal V, Guo H and Bartel DP: Expanded identification and characterization of mammalian circular RNAs. Genome Biol 15: 409, 2014.

6. Li Y, Zheng Q, Bao C, Li S, Guo W, Zhao J, Chen D, Gu J, He X and Huang S: Circular RNA is enriched and stable in exosomes: A promising biomarker for cancer diagnosis. Cell Res 25: 981-984, 2015.

7. Lux S and Bullinger L: Circular RNAs in cancer. Adv Exp Mol Cancer 1087: 215-230, 2018.

8. Zhang M and Xin Y: Circular RNAs: A new frontier for cancer diagnosis and therapy. J Hematol Oncol 11: 21, 2018.

9. Sang M, Meng L, Liu S, Ding P, Chang S, Ju Y, Liu F, Gu L, Lian Y and Geng C: Circular RNA ciRS-7 maintains metastatic phenotypes as a ceRNA of miR-1299 to target MMPs. Mol Cancer Res 16: 1665-1675, 2018.

10. Wei H, Pan L, Tao D and Li R: Circular RNA circZFR contributes to papillary thyroid cancer cell proliferation and invasion by sponging miR-1261 and facilitating C8orf4 expression. Biochem Biophys Res Commun 503: 56-61, 2018.

11. Zhong Y, Du Y, Yang X, Mo Y, Fan C, Xiong F, Ren D, Ye X, Li C Wang Y, et al: Circular RNAs function as ceRNAs to regulate and control human cancer progression. Mol Cancer 17: 79, 2018.

12. Huang L, Chen M, Pan J and Yu W: Circular RNA circNASP modulates the malignant behaviors in osteosarcoma via miR-1253/FOXF1 pathway. Biochem Biophys Res Commun 500: 511-517, 2018.

13. Yanbin Z and Jing Z: CircSAMD4A accelerates cell proliferation of osteosarcoma by sponging miR-1244 and regulating MDM2 mRNA expression. Biochem Biophys Res Commun 516: 102-111, 2019.

14. Qi W, Liang W, Jiang H and Miuyee Waye M: The function of miRNA in hepatic cancer stem cell. Biomed Res Int 2013: 358902, 2013.

15. Chi SW, Zang JB, Mele A and Darnell RB: Ago HITS-CLIP decodes miRNA-mRNA interaction maps. Nature 460: 479-486, 2009.

16. Allantaz F, Cheng DT, Bergauer T, Ravindran P, Rossier MF, Ebeling M, Badi L, Reis B, Bitter H, D'Asaro M, et al: Expression profiling of human immune cell subsets identifies miRNA-mRNA regulatory relationships correlated with cell type specific expression. PLoS One 7: e29979, 2012.

17. Althoff K, Lindner S, Odersky A, Mestdagh P, Beckers A Karczewski S, Molenaar JJ, Bohrer A, Knauer S, Speleman F, et al: miR-542-3p exerts tumor suppressive functions in neuroblastoma by downregulating Survivin. Int J Cancer 136: 1308-1320, 2015.

18. Chen Y, Han X, Yin X, Zhou Y and Wu T: Decreased expression of miR-132 in CRC tissues and its inhibitory function on tumor progression. Open Life Sci 11: 130-135, 2016.

19. Liu W, Kang L, Han J, Wang Y, Shen C, Yan Z, Tai Y and Zhao C: miR-342-3p suppresses hepatocellular carcinoma proliferation through inhibition of IGF-1R-mediated Warburg effect. Onco Targets Ther 11: 1643-1653, 2018.

20. Chen $\mathrm{H}$ and Tian Y: MiR-15a-5p regulates viability and matrix degradation of human osteoarthritis chondrocytes via targeting VEGFA. Biosci Trends 10: 482-488, 2017.

21. Chang SW, Yue J, Wang BC and Zhang XL: miR-503 inhibits cell proliferation and induces apoptosis in colorectal cancer cells by targeting E2F3. Int J Clin Exp Pathol 8: 12853-12860, 2015.

22. Hussein NA, Kholy ZA, Anwar MM, Ahmad MA and Ahmad SM: Plasma miR-22-3p, miR-642b-3p and miR-885-5p as diagnostic biomarkers for pancreatic cancer. J Cancer Res Clin Oncol 143: 83-93, 2017.

23. Kong XY, Du YQ, Li L, Liu JQ, Wang GK, Zhu JQ, Man XH, Gong YF, Xiao LN, Zheng YZ, et al: Plasma miR-216a as a potential marker of pancreatic injury in a rat model of acute pancreatitis. World J Gastroenterol 16: 4599-4604, 2010.

24. Liu BR and Xie L: microRNA: A new cancer biomarker. Chin Clin Oncol 15: 1-5, 2010 (In Chinese)

25. Li Q, Song S, Ni G, Li Y and Wang X: Serum miR-542-3p as a prognostic biomarker in osteosarcoma. Cancer Biomark 21: $521-526,2017$.
26. Tao K, Yang J, Guo Z, Hu Y, Sheng $\mathrm{H}$, Gao $\mathrm{H}$ and $\mathrm{Yu} \mathrm{H}$ : Prognostic value of miR-221-3p, miR-342-3p and miR-491-5p expression in colon cancer. Am J Transl Res 6: 391-401, 2014.

27. Wang D, Fan Z, Liu F and Zuo J: Hsa-miR-21 and Hsa-miR-29 in tissue as potential diagnostic and prognostic biomarkers for gastric cancer. Cell Physiol Biochem 37: 1454-1462, 2015.

28. Livak KJ and Schmittgen TD: Analysis of relative gene expression data using real-time quantitative PCR and the 2(-Delta Delta C(T)) method. Methods 25: 402-408, 2001

29. Li C, Wang F, Wei B, Wang L and Kong D: LncRNA AWPPH promotes osteosarcoma progression via activation of Wnt/ $\beta$-catenin pathway through modulating miR-93-3p/FZD7 axis. Biochem Biophys Res Commun 514: 1017-1022, 2019.

30. Yilmaz M and Christofori G: EMT, the cytoskeleton, and cancer cell invasion. Cancer Metastasis Rev 28: 15-33, 2009.

31. Jin $H$, Jin $X$, Zhang $H$ and Wang W: Circular RNA hsa-circ-0016347 promotes proliferation, invasion and metastasis of osteosarcoma cells. Oncotarget 8: 25571-25581, 2017.

32. Jin J, Chen A, Qiu W, Chen Y, Li Q, Zhou X and Jin D: Dysregulated circRNA_100876 suppresses proliferation of osteosarcoma cancer cells by targeting microRNA-136. J Cell Biochem 120: 15678-15687, 2019.

33. Pan Z, Sun X, Shan H, Wang N, Wang J, Ren J, Feng S, Xie L, Lu C, Yuan Y, et al: MicroRNA-101 inhibited postinfarct cardiac fibrosis and improved left ventricular compliance via the FBJ osteosarcoma oncogene/transforming growth factor- $\beta 1$ pathway. Circulation 126: 840-850, 2012.

34. Liu X, Zhong Y, Li J and Shan A: Circular RNA circ-NT5C2 acts as an oncogene in osteosarcoma proliferation and metastasis through targeting miR-448. Oncotarget 8: 114829-114838, 2017.

35. Cheng D, Fan J, Ma Y, Zhou Y, Qin K, Shi M and Yang J: LncRNA SNHG7 promotes pancreatic cancer proliferation through ID4 by sponging miR-342-3p. Cell Biosci 9: 28, 2019.

36. Gao Y, Zhang SG, Wang ZH and Liao JC: Down-regulation of miR-342-3p in hepatocellular carcinoma tissues and its prognostic significance. Eur Rev Med Pharmacol Sci 21: 2098-2102, 2017.

37. Hu K, Mu X, Kolibaba H, Yin Q, Liu C, Liang X and Lu J: Metadherin is an apoptotic modulator in prostate cancer through miR-342-3p regulation. Saudi J Biol Sci 25: 975-981, 2018.

38. Jones D, Anene D, Aloway A, Anene P, Avila D, Gobejishvili L, Barve S, Mcnally L and Kidd LC: Abstract 1844: Reduced expression of miR-342-3p in prostate cancer. Cancer Res 73: 1844-1844, 2013

39. Zhang S, Liu L, Lv Z,Li Q, Gong W and Wu H: MicroRNA-342-3p inhibits the proliferation, migration, and invasion of osteosarcoma cells by targeting astrocyte-elevated gene-1 (AEG-1). Oncol Res 25: 1505-1515, 2017

40. Asad M, Wong MK, Tan TZ, Choolani M, Low J, Mori S, Virshup D, Thiery JP and Huang RY: FZD7 drives in vitro aggressiveness in Stem-A subtype of ovarian cancer via regulation of non-canonical Wnt/PCP pathway. Cell Death Dis 5: e1346, 2014.

41. Deng B, Zhang Y, Zhang S, Wen F, Miao Y and Guo K: MicroRNA-142-3p inhibits cell proliferation and invasion of cervical cancer cells by targeting FZD7. Tumor Biol 36: 8065-8073, 2015.

42. Kirikoshi H, Sekihara $\mathrm{H}$ and Katoh M: Up-regulation of Frizzled-7 (FZD7) in human gastric cancer. Int J Oncol 19: 111-115, 2001

43. Qiu X, Jiao J, Li Y and Tian T: Overexpression of FZD7 promotes glioma cell proliferation by upregulating TAZ. Oncotarget 7: 85987-85999, 2016.

44. Cao TT, Xiang D, Liu BL, Huang TX, Tan BB, Zeng CM, Wang ZY, Ming XY, Zhang LY, Jin G, et al: FZD7 is a novel prognostic marker and promotes tumor metastasis via WNT and EMT signaling pathways in esophageal squamous cell carcinoma. Oncotarget 8: 65957-65968, 2017.

45. Cheng ZX, Song YX, Wang ZY, Wang Y and Dong Y: miR-144-3p serves as a tumor suppressor by targeting FZD7 and predicts the prognosis of human glioblastoma. Eur Rev Med Pharmacol Sci 21: 4079-4086, 2017.

46. Chen Z, Huang C, Ma T, Jiang L, Tang L, Shi T, Zhang S, Zhang L, Zhu P, Li J and Shen A: Reversal effect of quercetin on multidrug resistance via FZD7/ $\beta$-catenin pathway in hepatocellular carcinoma cells. Phytomedicine 43: 37-45, 2018.

This work is licensed under a Creative Commons Attribution-NonCommercial-NoDerivatives 4.0 International (CC BY-NC-ND 4.0) License. 